\title{
Periodic orbit theory for the Hénon-Heiles system in the continuum region
}

\author{
J. Kaidel, P. Winkler ${ }^{1}$ and M. Brack \\ Institut für Theoretische Physik, Universität Regensburg, D-93040 Regensburg, Germany \\ ${ }^{1}$ Department of Physics, University of Nevada Reno, NV 89557, USA
}

(September 27, 2018)

\begin{abstract}
We investigate the resonance spectrum of the Hénon-Heiles potential up to twice the barrier energy. The quantum spectrum is obtained by the method of complex coordinate rotation. We use periodic orbit theory to approximate the oscillating part of the resonance spectrum semiclassically and Strutinsky smoothing to obtain its smooth part. Although the system in this energy range is almost chaotic, it still contains stable periodic orbits. Using Gutzwiller's trace formula, complemented by a uniform approximation for a codimension-two bifurcation scenario, we are able to reproduce the coarse-grained quantum-mechanical density of states very accurately, including only a few stable and unstable orbits.
\end{abstract}

05.45.Mt,03.65.Sq

\section{INTRODUCTION}

Understanding the way in which scattering resonances modify the density of states in the continuum region of a quantum-mechanical system has been a challenging problem since the early days of quantum mechanics [1]. Processes involving quantum resonances are ubiquitous in nature and technology [2]. They contribute, e.g., to the conductance fluctuations in transport phenomena [3]. The focus of our present paper lies on the semiclassical evaluation of the density of states of an open system. The periodic orbit theory developed over 35 years ago by Gutzwiller [4], and its extension to closed orbits [5], have had an enormous impact on the recent research on "quantum chaos", i.e., the study of quantum signatures of classical chaos [6-8]. Numerous studies have shown that resonance spectra can also be approximated semiclassically, using either the closed or the periodic orbits of the underlying classical system. Besides efforts which were limited to fully chaotic systems $[9,10]$, recent interest has focused on general systems with mixed phase-space dynamics, including potentials which do not vanish asymptotically [11-13]. However, a satisfactory semiclassical description of resonance spectra could only be achieved in those limits where all orbits are unstable and can be completely enumerated by symbolic dynamics. Truly mixed-dynamical systems, in which unstable and stable orbits coexist and undergo bifurcations, pose serious problems for the semiclassical theories. Although the divergences arising at bifurcations can be remedied by uniform approximations for the simplest codimension-one $[14,15]$ and codimension-two scenarios [16-18], their enormous proliferation with increasing orbit length renders a semiclassical determination of the fine structure of quantum spectra practically impossible.

Presently we study the two-dimensional Hénon-Heiles (HH) potential [19] which is a well-known paradigm for a mixed system [20] and has served as a model for various physical systems of different nature [21-24]. This paper is the sequel of a recent semiclassical study of the HH potential up to the barrier energy [18]. Here we shall determine the quantum-mechanical resonance spectrum above the barrier, extract the smooth density of states by Strutinsky averaging [26], and calculate its oscillating part via Gutzwiller's trace formula [4], incorporating a uniform approximation to regularize a codimension-two sequence of periodic orbit bifurcations.

While the fine structure of the spectrum is not accessible semiclassically for the reasons stated above, we consider here the coarse-grained density of states obtained by a Gaussian convolution over a finite energy range. This allows one to include only a finite number of shorter orbits but still to reproduce the gross-shell structure of the level density [25], as has been exemplified in various models and physical applications (see, e.g., [27]). For the HH potential, this was shown in the low-energy range [28] using a uniform treatment of the SU(2) symmetry limit, and for energies close to the barrier [18] using a uniform treatment of sequences of pitchfork bifurcations. Here we show that also in the continuum region above the barrier, the coarse-grained quantum-mechanical density of states is very well reproduced semiclassically using a relatively small number of unstable and stable periodic orbits. 


\section{DENSITY OF STATES INCLUDING THE CONTINUUM}

Let us consider a particle scattered by a spherically symmetric one-body potential $V(r)<0$ with $V(r) \rightarrow 0$ for $r \rightarrow \infty$. The density of states of the free system in the continuum region, given by

$$
g_{f r e e}(E)=c \sqrt{E} \quad(E>0)
$$

with constant $c$, is modified through the scattering resonances by a contribution [1]

$$
\Delta g(E)=\frac{1}{\pi} \sum_{l=0}^{\infty} \frac{\partial \delta_{l}(E)}{\partial E} . \quad(E>0)
$$

Here $l$ are the quantum numbers of the orbital angular momentum and $\delta_{l}(E)$ is the elastic scattering phase shift of the $l$-th partial wave. By definition, resonances occur at those energies $E_{l}$ where the phase shift takes the value $\delta_{l}\left(E_{l}\right)=\pi / 2$. Expanding the phase shift around the resonance energy one obtains

$$
\delta_{l}(E)=\arctan \left(\frac{\Gamma_{l} / 2}{E-E_{l}}\right)+\ldots
$$

where $\Gamma_{l}$ is the width of the resonance, related to its life time $\tau_{l}$ by $\Gamma_{l}=\hbar / \tau_{l}$. Inserting (3) into (2), keeping only the leading term, leads to

$$
\Delta g(E)=\frac{1}{\pi} \sum_{l=0}^{\infty} \frac{\Gamma_{l} / 2}{\left(E-E_{l}\right)^{2}+\left(\Gamma_{l} / 2\right)^{2}}
$$

In the region $E<0$, where the potential has only discrete eigenvalues $E_{n l}$ with a radial quantum number $n$, the density of states is given by a sum of delta functions and the total density of states for the system is given by

$$
g_{\text {tot }}(E)=g_{\text {free }}(E)+\sum_{n, l=0}^{\infty} \delta\left(E-E_{n l}\right)+\Delta g(E) .
$$

For a non-integrable system without spherical symmetry, the spectrum of both bound states and resonances can only be characterized by one quantum number, say $m$, replacing $(n, l)$ in the above. We thus rewrite (4) as

$$
\Delta g(E)=g_{\text {tot }}(E)-g_{\text {free }}(E)=\frac{1}{\pi} \sum_{m} \frac{\Gamma_{m} / 2}{\left(E-E_{m}\right)^{2}+\left(\Gamma_{m} / 2\right)^{2}}
$$

whereby the bound spectrum is automatically included since the Lorentzians on the r.h.s. go over into delta functions for $\Gamma_{m} \rightarrow 0$.

We next define a coarse-grained density of states, performing a Gaussian convolution of (6) over an energy range $\gamma$. This can be done analytically, leading to

$$
\Delta g_{\gamma}(E)=\frac{1}{\gamma \sqrt{\pi}} \int_{-\infty}^{\infty} \Delta g\left(E^{\prime}\right) e^{-\left(E-E^{\prime}\right)^{2} / \gamma^{2}} \mathrm{~d} E^{\prime}=\frac{1}{\gamma \sqrt{\pi}} \sum_{m} \Re e\left[w\left(z_{m}\right)\right]
$$

with

$$
w(z)=e^{-z^{2}} \operatorname{erfc}(-i z), \quad z_{m}=\frac{\left(E_{m}+i \Gamma_{m} / 2-E\right)}{\gamma},
$$

where $\operatorname{erf}(z)=1-\operatorname{erfc}(z)$ is the error function [29]. 


\section{NUMERICAL CALCULATION OF THE HÉNON-HEILES SPECTRUM}

For a general potential $V(\mathbf{r})$ without spherical symmetry, it may become difficult to calculate the scattering phase shifts. Furthermore, if the potential has a continuous spectrum above some threshold energy $E_{t h}$ but does not reach $E_{t h}$ asymptotically for $r \rightarrow \infty$ (such as the Hénon-Heiles potential considered below), there are generally no free plane-wave solutions for $E>E_{t h}$ and the phase shifts cannot be defined. Nevertheless, there are ways to calculate complex resonance energies $E_{m}^{*}=E_{m}-i \Gamma_{m} / 2$ which appear as poles of the Green function in the complex energy plane $E^{*}$ with $\Re e E^{*}>E_{t h}$.

One convenient way to obtain the resonances without requiring the knowledge of phase shifts is given by the method of complex rotation [30-33]. Here one solves the scaled Schrödinger equation

$$
\left[\hat{S} \hat{H}(\mathbf{r}) \hat{S}^{-1}\right] \hat{S} \phi_{m}^{r e s}(\mathbf{r})=\left(E_{m}-i \Gamma_{m} / 2\right) \hat{S} \phi_{m}^{r e s}(\mathbf{r})
$$

where $\hat{S}$ is the similarity transformation (or complex rotation)

$$
\hat{S} f(\mathbf{r})=f\left(\mathbf{r} e^{i \theta}\right)
$$

which multiplies each spatial coordinate of an analytical function $f(\mathbf{r})$ by a complex exponential with real phase $\theta$. This transformation turns a resonance wavefunction $\phi_{m}^{r e s}(\mathbf{r})$ into a square-integrable function which can be expanded in Hilbert space [33]. For systems with asymptotically free states, the energies $E>E_{t h}$ of all nonresonant continuum states are rotated in the complex plane to the line $\left(E-E_{t h}\right) \exp (-2 i \theta)$, whereas the poles at $E_{m}^{*}=E_{m}-i \Gamma_{m} / 2$ corresponding to the resonant states remain independent of $\theta$, provided that this angle is large enough to "uncover" the poles, i.e., $2 \theta>\arctan \left[\Gamma_{m} / 2\left(E_{m}-E_{t h}\right)\right]$. Practical experience shows [34] that $\theta$-independent poles can also be found if the non-resonant continuum states are not asymptotically free, although this has not been proven rigorously. Note that the discrete eigenenergies in the bound region $E<E_{t h}$ are also obtained by the complex rotation method; they stay on the real energy axis with $E<E_{t h}$ and have zero imaginary parts.

Having determined the energies $E_{m}^{*}=E_{m}-i \Gamma_{m} / 2$, including the bound spectrum with $\Gamma_{m}=0$, their contribution to the density of states is given by

$$
\Delta g(E)=-\frac{1}{\pi} \Im m \sum_{m} \frac{1}{E-E_{m}+i \Gamma_{m} / 2}
$$

leading to (6).

We now want to investigate the density of states of the two-dimensional Hénon-Heiles (HH) Hamiltonian

$$
\hat{H}=-\frac{1}{2}\left(\hat{p}_{x}^{2}+\hat{p}_{y}^{2}\right)+\frac{1}{2}\left(x^{2}+y^{2}\right)+\alpha\left(x^{2} y-y^{3} / 3\right),
$$

with $\alpha>0$ and units such that $\hbar=1$. This Hamiltonian describes an open system in which a particle can escape by direct transmission over - or by tunneling through - one of three barriers with height $E_{b a r}=1 / 6 \alpha^{2}$. However, since the potential goes asymptotically to $-\infty$ like $-r^{3}$ (with $r^{2}=x^{2}+y^{2}$ ) in some regions of space, the system has no discrete eigenstates and we must put $E_{t h}=-\infty$. For sufficiently small values of the parameter $\alpha$, there are quasi-bound states below the three barriers, but they have finite widths due to tunneling.

Scaling both coordinates and momenta with the factor $1 / \alpha$ causes the classical dynamics to depend only on the scaled energy $e=E / E_{b a r}=6 \alpha^{2} E$; the barrier energy then lies at $e_{b a r}=1$. In the following we present all real parts of the spectrum in the scaled energy units $e$.

To solve the complex Schrödinger equation (9) for the system (12), we diagonalize it in a truncated basis $\{|n m\rangle\}$ of the two-dimensional isotropic harmonic oscillator with $n, m \leq N$. This leads to the eigenvalue problem for the complex non-Hermitian matrix

$$
\begin{aligned}
{[H(\theta)]_{n^{\prime} m^{\prime} n m} } & \equiv\left\langle n^{\prime} m^{\prime}\left|\hat{S} \hat{H}(\mathbf{r}) \hat{S}^{-1}\right| n m\right\rangle \\
& =T_{n^{\prime} m^{\prime} n m} e^{-2 i \theta}+\left(V_{H O}\right)_{n^{\prime} m^{\prime} n m} e^{2 i \theta}+\alpha\left(V_{3}\right)_{n^{\prime} m^{\prime} n m} e^{3 i \theta}
\end{aligned}
$$

The matrices $T_{n^{\prime} m^{\prime} n m},\left(V_{H O}\right)_{n^{\prime} m^{\prime} n m}$ and $\left(V_{3}\right)_{n^{\prime} m^{\prime} n m}$ are the real matrices of the kinetic, harmonic and cubic parts of (12), respectively, for $\theta=0$. The complex eigenvalues $E_{m}^{*}=E_{m}-i \Gamma_{m}$ of the matrix (13) were found numerically using its sparse property. 


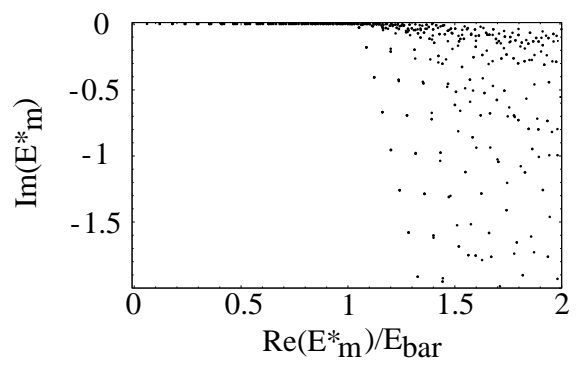

FIG. 1. Resonances $E_{m}^{*}=E_{m}-i \Gamma_{m}$ of the Hénon-Heiles system with $\alpha=0.1$ : imaginary parts versus real parts (the latter in scaled energy units $\left.e=E / E_{b a r}\right)$.

In Fig. 1 the complex resonance spectrum of the HH system (12) for $\alpha=0.1$ is shown. The size of the truncated basis $|n m\rangle$ was given by $N=n_{\max }=m_{\max }=130$. We only give the spectrum above the minimum of the classically bound region, located at $e=0$. Due to the truncation of the basis in Hilbert space, it is known [32] that the poles in the complex energy plane slightly depend on the rotation angle $\theta$; their optimal values are then found as stationary points (or plateau values) with respect to small variations of $\theta$. We could determine the plateau values of the resonances with an accuracy of 6 digits over an interval in $\theta$ of about 10 degrees, as is shown by the example in Fig. 2. (The real part of the resonance, $E_{m} / E_{\text {bar }}=0.928966$, is constant within 6 digits in the whole interval of $\theta$ shown.) Note that the imaginary parts of the quasi-bound states for $e<1$ are exponentially small except very near the barriers. For the states slightly above the barriers, a semiclassical prediction of the imaginary parts, which is in good agreement with our numerical results, was given in [35]. The quasi-regular pattern observed in the region $e>1$, where some of the resonances lie on almost parallel "rays" in the complex energy plane, is a reminiscence of the separable system that is obtained if one neglects the coupling term $\alpha x^{2} y$ in (12) (see Ref. [18] for the density of states of this separable system).

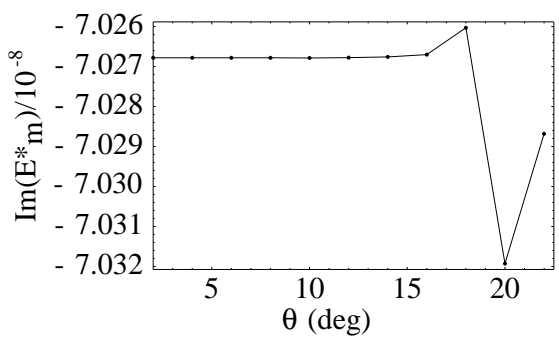

FIG. 2. Imaginary part $-\Gamma_{m}$ of a resonance with real part $E_{m} / E_{b a r}=0.928966$, plotted versus the rotation angle $\theta$.

\section{SMOOTH PART OF DENSITY OF STATES}

The main purpose of our paper is to establish the classical-to-quantum correspondence, approximating the density of states (6) by a semiclassical trace formula [4]. As usual, the trace formula only yields the oscillating part $\delta g_{\gamma}(E)$ of the total density of states, written as

$$
g_{\text {tot }}(E)=g_{\text {free }}+\widetilde{g}(E)+\delta g_{\gamma}(E)
$$

where $g_{\text {free }}$ in the present two-dimensional system is a constant. The function $\widetilde{g}(E)$ is the smoothly varying nonperiodic part of (6), which usually is obtained from the (extended) Thomas-Fermi (ETF) model [27]. In the present HH system, however, we have the problem that the ETF level density cannot be defined for $e>1$ where the system is open. We therefore resort to the numerical Strutinsky averaging [26] which is equivalent to the ETF model where the latter can be used [27]. As shown in [36], the Strutinsky averaging corresponds to approximating the average part of the density of states near the energy $E$ by a polynomial of given power $2 s$, i.e., a truncated Taylor expansion with $s=1,2, \ldots$ In practice it is obtained by convolution of the density of states with a Gaussian of width $\tilde{\gamma}$, modified by a suitable linear combination of Hermite polynomials up to order $2 s$. The smoothing function can also be compactly written as [37] 


$$
f_{\tilde{\gamma}, s}(E)=\frac{1}{\tilde{\gamma} \sqrt{\pi}} e^{-(E / \tilde{\gamma})^{2}} L_{s}^{1 / 2}\left[(E / \tilde{\gamma})^{2}\right]
$$

where $L_{s}^{1 / 2}$ is an associated Laguerre polynomial. The average part of (6) is then obtained by the convolution

$$
\widetilde{g}(E)=\int_{-\infty}^{\infty} \Delta g\left(E^{\prime}\right) f_{\tilde{\gamma}, s}\left(E-E^{\prime}\right) \mathrm{d} E^{\prime} .
$$

Ideally, the results obtained in this way will not depend on $s$ and $\tilde{\gamma}$, provided that $\tilde{\gamma}$ is chosen to be larger than the characteristic energy spacing of the main shells in the spectrum and $s$ is large enough. This is indeed the case if the true average (ETF) density of states is a polynomial of order $\leq 2 s$. Practically, one has to look for stationary values of the results as functions of both $\tilde{\gamma}$ and $s$, fulfilling the so-called "plateau condition" [36]. The integral in (16), with $\Delta g(E)$ given by (6), can be calculated analytically (see Appendix).

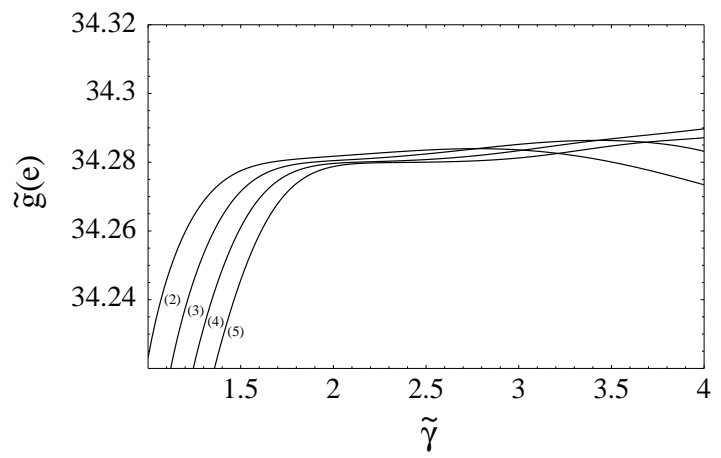

FIG. 3. Test of the plateau condition for the average density of states of the HH system with $\alpha=0.1$, evaluated at the energy $e=1.5$. The numbers in brackets give the order $s$ of the Laguerre polynomial in (15).

In Fig. 3 we show the plateaux in $\widetilde{g}(E)$ obtained by varying $\tilde{\gamma}$ and the polynomial order $s$ at the fixed energy $e=1.5$. One can see that the stationary condition is reasonably well fulfilled for $\tilde{\gamma} \approx 2.2$, independently of $s>2$. Plateaux of this quality have been obtained for the spectra of finite-depth Woods-Saxon potentials appropriate for nuclear physics, and the plateau values of the averaged quantitities have been shown to be identical with their ETF values within the numerical accuracies [38].

Having determined the optimal plateau values at all energies of interest, the quantum-mechanical value of $\delta g_{\gamma}(E)$ is given by

$$
\delta g_{\gamma}(E)=g_{\gamma}(E)-\widetilde{g}(E) .
$$

In the HH system, the function $\widetilde{g}(E)$ varies rather abruptly near $e=1$ on a scale comparable to the oscillations of $\delta g_{\gamma}(E)$, so that no ideal plateaux are found and there remains a small numerical uncertainty near $e \gtrsim 1$.

\section{SEMICLASSICAL CALCULATION OF THE COARSE-GRAINED RESONANCE SPECTRUM}

Next we want to construct the semiclassical approximation of $\delta g_{\gamma}(E)$ in the form of Gutzwiller's trace formula for isolated orbits [4], modified by the exponential factor which is the result of the coarse-graining over the energy range $\gamma$ and suppresses the contributions from orbits with longer periods:

$$
\delta g_{s c l}(E)=\frac{1}{\pi \hbar} \sum_{\xi} \frac{T_{\xi}(E)}{r_{\xi} \sqrt{\left|\operatorname{Tr} \widetilde{M}_{\xi}(E)-2\right|}} e^{-\left[\gamma T_{\xi}(E) / 2 \hbar\right]^{2}} \cos \left[\frac{S_{\xi}(E)}{\hbar}-\frac{\pi}{2} \sigma_{\xi}\right],
$$

The sum goes over all isolated periodic orbits labeled by $\xi$, and the other quantities in (18) are, as usual, the periods $T_{\xi}$ and actions $S_{\xi}$, the Maslov indices $\sigma_{\xi}$ and the repetition numbers $r_{\xi}$ of the periodic orbits. $\widetilde{M}_{\xi}(E)$ are the stability matrices obtained by linearization of the equations of motion along the periodic orbits.

The shortest periodic orbits of the classical HH system (12) were obtained using a numerical Newton-Raphson algorithm [39]. They have already been extensively studied in earlier papers [40-44]. We use here the nomenclature introduced in [44], where the Maslov indices $\sigma_{\xi}$ appear as subscripts in the symbols $\left(\mathrm{B}_{4}, \mathrm{R}_{5}, \mathrm{~L}_{6}\right.$, etc.) of the orbits. The Maslov indices were obtained by the method developed in [45] (see Ref. [27] for practical recipes). 


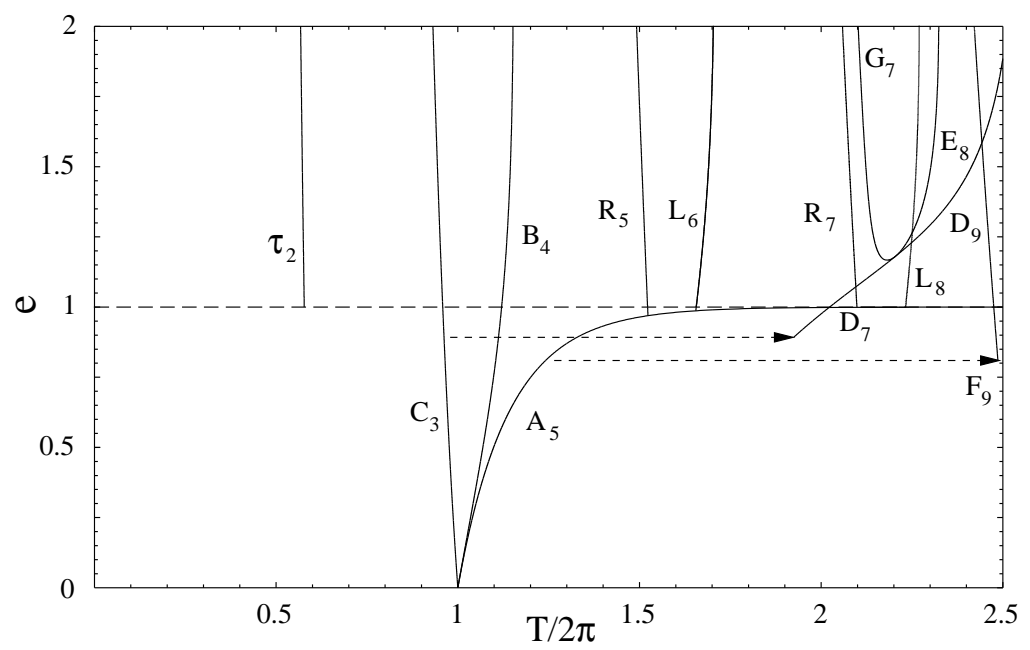

FIG. 4. Scaled energy $e$ versus periods $T_{\xi} / 2 \pi$ of the shortest periodic orbits in the HH potential. The dotted arrows correspond to period-doubling bifurcations.

Fig. 4 shows the periods of the shortest periodic orbits as functions of the scaled energy $e$ of the system up to twice the barrier energy. One can see that there still exist many periodic orbits above the barrier energy where the particle has enough energy to escape from the bound region. Actually, there is an infinite number of orbits of type R and L (only the two shortest of each are shown here), born from the saddle-line orbit A in a cascade of bifurcations [41,43,44] cumulating at $e=1$. They exist at all energies $e$ above their respective bifurcations but become very unstable at higher energies. Above the barriers $(e>1$ ), new orbits (named $\mathrm{S}$ in [41] and $\tau$ in [42,43]) with Maslov index 2 arise, librating across the saddles. Although these $\tau_{2}$ orbits are quite unstable, they have the smallest periods of all orbits and therefore play an important role for the coarse-grained density of states at $e>1$, as discussed in the following.

Here we concentrate on the density of states above the barriers $(e \geq 1)$. For semiclassical calculations at $e<1$, we refer to earlier papers $[18,28]$. The periodic orbits in the region $e>1$ are not all unstable. This can be inferred from Fig. 4 for the bifurcation of the stable orbit $\mathrm{D}_{7} / \mathrm{D}_{9}$ near $e \simeq 1.18$, in which the orbit $\mathrm{E}_{8}$ and, indirectly, also the orbit $\mathrm{G}_{7}$ is involved. It is seen directly in Fig. 5, where we show the traces of their stability matrices versus $e$.

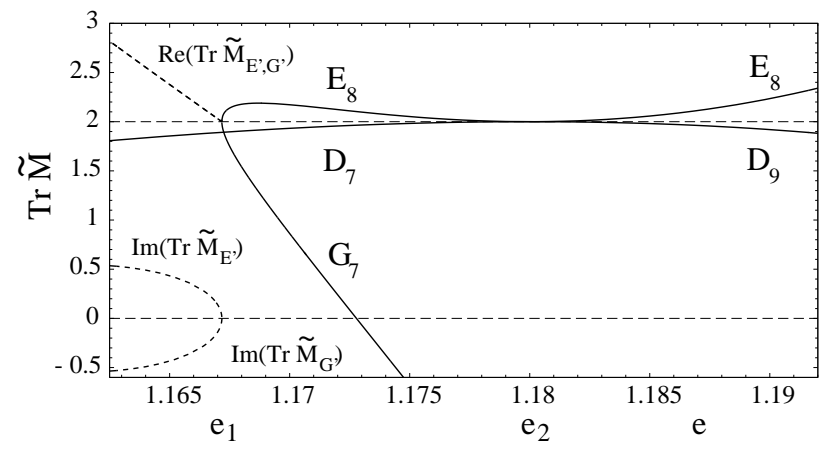

FIG. 5. Trace of stability matrix $\widetilde{M}_{\xi}$ vs. scaled energy $e$ for the period-two orbits $\xi=\mathrm{D}_{7} / \mathrm{D}_{9}, \mathrm{E}_{8}$, and $\mathrm{G}_{7}$ taking part in a codimension-two sequence of bifurcations. The short-dashed lines show the values for the ghost orbits $\mathrm{E}^{\prime}$ and G' associated to the tangent bifurcation. (Upper left: common real part, lower left: imaginary parts of $\operatorname{tr} \widetilde{M}_{E^{\prime}} \operatorname{and} \operatorname{tr} \widetilde{M}_{G^{\prime}}$.)

In Fig. 6 we show the shapes of these three orbits at three energies around $e \simeq 1.18$ where the $\mathrm{D}$ and $\mathrm{E}$ orbits meet. Note that the central $\mathrm{D}$ orbit (solid lines) has $C_{3}$ symmetry. The satellite orbits $\mathrm{E}_{8}$ (dashed lines) and $\mathrm{G}_{7}$ (dash-dotted lines) do not have this symmetry and occur in three orientations (and hence must be included thrice in the trace formula). All three orbits have an additional degeneracy of two from time reversal symmetry.

This scenario represents a sequence of bifurcations corresponding to an unfolding of codimension two: at $e_{1}=$ 1.16717, the orbits $\mathrm{E}_{8}$ and $\mathrm{G}_{7}$ are born in a tangent bifurcation; then the unstable orbit $\mathrm{E}_{8}$ and the stable orbit $\mathrm{D}_{7}$ meet at $e_{2}=1.18000$ in a touch-and-go bifurcation, leaving it as $\mathrm{D}_{9}$ and $\mathrm{E}_{8}$. The two bifurcations are too close to be treated separately as codimension-one scenarios, because the action differences of the orbits involved between the two bifurcation energies, $\Delta S_{\xi}=S_{\xi}\left(e_{2}\right)-S_{\xi}\left(e_{1}\right)$, do not fulfill the requirement $\left|\Delta S_{\xi}\right| \gg \hbar$. 

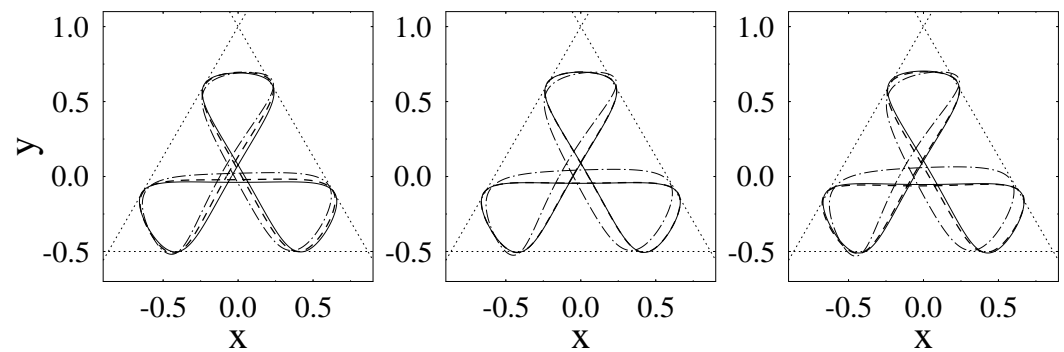

FIG. 6. Shapes of orbits $\mathrm{D}_{7} / \mathrm{D}_{9}$ (solid), $\mathrm{E}_{8}$ (dashed), and $\mathrm{G}_{7}$ (dash-dotted lines) in the $(x, y)$ plane, shown at the three energies $e=1.170$ (left panel), $e=1.179$ (center panel), and $e=1.190$ (right panel). The dotted lines are the equipotential lines at $e=1$ intersecting at the three saddle points.

Since the Gutzwiller trace formula (18) diverges at the bifurcation points, so-called uniform approximations [14,15] must be used. For the codimension-two scenario described above, a suitable uniform approximation has been developed by Schomerus [17]; we have adapted it to the present scenario (see [39] for the technical details). Expanding the normal form used in the trace integral near the two bifurcations [17], the traces of the stability matrices of the participating orbits are given by

$$
\operatorname{tr} \widetilde{M}_{\mathrm{E}, \mathrm{G}}=2 \mp 6 a \sqrt{-\epsilon_{1} / 3 a}-\frac{8 b}{3 a} \epsilon_{1}+\mathcal{O}\left(\left|\epsilon_{1}\right|^{3 / 2}\right),
$$

with $\epsilon_{1}=c_{1}\left(e-e_{1}\right)$ near the first bifurcation, and by

$$
\begin{aligned}
& \operatorname{tr} \widetilde{M}_{\mathrm{D}}=2-\epsilon_{2}^{2}, \\
& \operatorname{tr} \widetilde{M}_{\mathrm{E}}=2+3 \epsilon_{2}^{2}+\mathcal{O}\left(\epsilon_{2}^{4}\right),
\end{aligned}
$$

with $\epsilon_{2}=c_{2}\left(e-e_{2}\right)$ near the second bifurcation. The behaviour of $\operatorname{tr} \widetilde{M}_{\xi}(e)$ given by the above formulae near the two bifurcations, i.e. for $\epsilon_{1} \ll 1$ and $\epsilon_{2} \ll 1$, can clearly be recognized in Fig. 5, where the solid lines represent the real parts of $\operatorname{tr} \widetilde{M}_{\xi}(e)$. The short-dashed lines shown for $e \leq e_{1}$ represent the real part (upper left) and imaginary parts (lower left) of $\operatorname{tr} \widetilde{M}_{E^{\prime}, G^{\prime}}(e)$ corresponding to the complex continuations of the periodic orbits $\mathrm{E}_{8}$ and $\mathrm{G}_{7}$ taking part in the tangent bifurcation, i.e., the so-called "ghost orbits", denoted here by E' and G'. They have to be included in order to obtain a continuous description of the semiclassical density of states throughout the whole bifurcation region. The parameters $a<0, b<0, c_{1}>0$, and $c_{2}>0$ appearing in Eqs. (19) and (20) come from the normal form of the trace integral, given in [17], and depend on the system. They need, however, not be determined explicitly but can be expressed in terms of the numerically calculated quantities $S_{\xi}$ and $\operatorname{tr} \widetilde{M}_{\xi}(e)$ of the periodic orbits $[17,39]$.

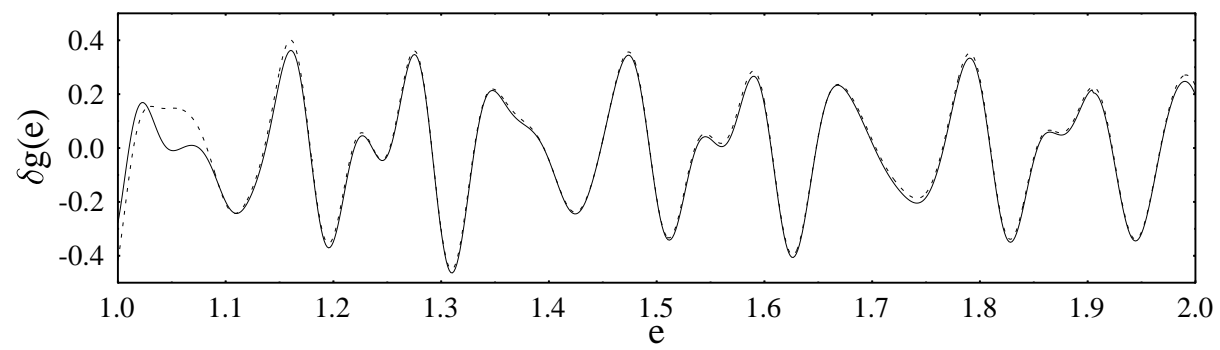

FIG. 7. Oscillating part of the coarse-grained density of states of the HH system with $\alpha=0.1$. The quantum result is shown by the solid, the semiclassical result by the dashed line. Gaussian smoothing range: $\gamma=0.5$. The primitive orbits $\mathrm{C}_{3}, \mathrm{~B}_{4}, \mathrm{R}_{5}$, $\mathrm{L}_{6}$, and the first three repetitions of $\tau_{2}$ are included in the trace formula (18).

In Fig. 7 we show a comparison between the quantum-mechanical and the semiclassical result for the oscillating part $\delta g_{\gamma}(E)$ of the coarse-grained density of states in the energy region $1 \leq e \leq 2$. Hereby an energy averaging width $\gamma=0.5$ has been used. In the semiclassical calculation, only the primitive orbits $\mathrm{C}_{3}, \mathrm{~B}_{4}, \mathrm{R}_{5}, \mathrm{~L}_{6}$, and the first three repetitions of $\tau_{2}$ had to be included for this energy resolution; no bifurcation occurs for these orbits so that the original trace formula for isolated orbits (18) could be used. The agreement is seen to be very good except in the region just 
above $e=1$. The small discrepancies seen there are due to the plateau uncertainties in the Strutinsky averaging of the smooth density of states, which have been mentioned at the end of Sec. IV.

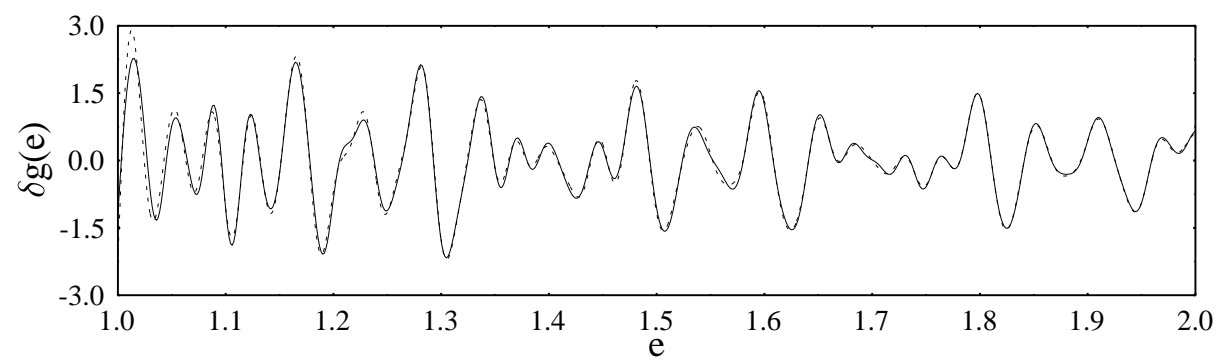

FIG. 8. Same as Fig. 7, but with Gaussian smoothing range $\gamma=0.25$. Here also $\mathrm{R}_{7}$ and $\mathrm{L}_{8}$, the second repetitions of $\mathrm{B}_{4}$ and $\mathrm{C}_{3}$, up to five repetitions of the orbit $\tau_{2}$, and the period-two orbits $\mathrm{D}_{7} / \mathrm{D}_{9}, \mathrm{E}_{8}$ and $\mathrm{G}_{7}$ are included; the latter three in the uniform approximation discussed in the text.

In Fig. 8 the same comparison is made, but this time with a finer energy resolution given by $\gamma=0.25$. To obtain convergence of the trace formula, also the period-two orbits $\mathrm{D}_{7} / \mathrm{D}_{9}, \mathrm{E}_{8}$ and $\mathrm{G}_{7}$ had to be included (in the uniform approximation mentioned above), besides the orbits $\mathrm{R}_{7}$ and $\mathrm{L}_{8}$, the second repetitions of $\mathrm{B}_{4}$ and $\mathrm{C}_{3}$, and up to five repetitions of the orbit $\tau_{2}$. Again the agreement between quantum mechanics and semiclassics is nearly perfect, in spite of the rather complex gross-shell structure in $\delta g(E)$. The uncertainties in $\widetilde{g}(E)$ here have less relative weight than for $\gamma=0.5$, due to the larger overall amplitude of the oscillations in $\delta g(E)$.

Finally, in Fig. 9 we show the results obtained with exactly the same parameters as in Fig. 8, but this time omitting the stable orbit $\mathrm{D}_{7} / \mathrm{D}_{9}$ and its companions $\mathrm{E}_{8}$ and $\mathrm{G}_{7}$ involved in the bifurcation. The difference to Fig. 8 is not large, but it shows that the inclusion of a stable bifurcating orbit in this mixed system does improve the semiclassical description.

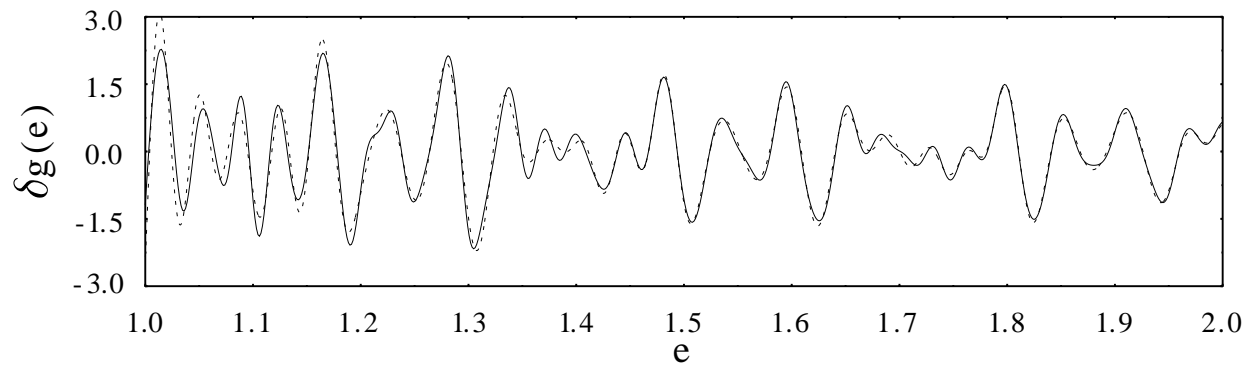

FIG. 9. Same as Fig. 8, but omitting the orbits D, E, and G participating in the bifurcations.

The process of diminishing $\gamma$ and including longer periodic orbits could, in principle, be continued - but at the cost of having to deal with more and more bifurcations, including scenarios of codimension higher than two. This just expresses the practical impossibility of obtaining full semiclassical quantisation in a mixed system, which we already discussed in the introduction. Here we have demonstrated, however, that the slightly coarse-grained density of states can be well described semiclassically using a relatively small number of periodic orbits, one of which $\left(\mathrm{D}_{7} / \mathrm{D}_{9}\right)$ is stable in a sizeable part of the energy region explored.

\section{SUMMARY AND CONCLUSIONS}

We have presented the first semiclassical calculation of the density of states in the Hénon-Heiles (HH) potential in the high-energy continuum region up to twice the barrier energy $(e=2)$. Above the barrier $(e>1)$, the density of states is dominated by resonances with appreciable imaginary parts. We have calculated the quantum-mechanical resonance spectrum using the method of complex rotation. Even though the HH system is classically almost chaotic for $e>1$, it still possesses small stable islands and therefore exhibits the general problems with the semiclassical 
quantization that are characteristic of mixed systems. We have shown, however, that the slightly coarse-grained density of states with a finite energy resolution $\gamma$ can very well be approximated semiclassically in terms of relatively few stable and unstable periodic orbits. For an energy resolution of $\gamma=0.25$ (see Fig. 8), we had to use only 11 orbits; three of them as a cluster taking part in a codimension-two bifurcation, and the rest as isolated orbits. The agreement between the semiclassical and the quantum-mechanical results for the oscillating part $\delta g_{\gamma}(E)$ of the density of states is excellent, except near the barrier energy $(e \gtrsim 1)$ where it is difficult to extract a unique average part $\widetilde{g}(E)$ of the density of states. While the (extended) Thomas-Fermi model fails completely, due to the unboundedness of the system for $e>1$, we have successfully implemented the numerical Strutinsky-averaging technique to obtain the average part. Some minor uncertainties in its plateau value remaining near $e \gtrsim 1$ play a decreasing relative role with increasing energy resolution, i.e., with decreasing coarse-graining width $\gamma$.

We conclude that the semiclassical trace formula, complemented by the uniform treatment of bifurcating periodic orbits, is a very economic tool for prediciting quantum oscillations also in the continuum region of a mixed-dynamical Hamiltonian system which is dominated by scattering resonances.

We are grateful to Gregor Tanner for a careful reading of the manuscript and for clarifying comments. This work was supported by the Deutsche Forschungsgemeinschaft (DFG) via the graduate college 638 "Nonlinearity and Nonequilibrium in Condensed Matter". One of the authors (P.W.) gratefully acknowledges travel support from the Office of Academic Affairs and the Foundation of the University of Nevada at Reno.

\section{APPENDIX: STRUTINSKY AVERAGING OF THE RESONANCE SPECTRUM}

The Strutinsky-averaged density of resonances is, according to Eqs. (6), (15) and (16), defined by

$$
\widetilde{g}(E)=\frac{1}{\tilde{\gamma} \sqrt{\pi}} \int_{-\infty}^{\infty} e^{-\left[\left(E-E^{\prime}\right) / \tilde{\gamma}\right]^{2}} L_{s}^{\frac{1}{2}}\left[\left(\frac{E-E^{\prime}}{\tilde{\gamma}}\right)^{2}\right] \frac{1}{\pi} \sum_{m} \frac{\Gamma_{m} / 2}{\left(E^{\prime}-E_{m}\right)^{2}+\left(\Gamma_{m} / 2\right)^{2}} \mathrm{~d} E^{\prime} .
$$

Without the Laguerre polynomial $L_{s}^{\frac{1}{2}}$, the integral in (21) is identical to that in (7) and can be expressed in terms of the quantities given in (8) with the substitution $\gamma \rightarrow \tilde{\gamma}$. The Laguerre polynomials with $s>0$ create even powers of $\left(E-E^{\prime}\right) / \tilde{\gamma}$ under the integral. These can be included using the formula

$$
\int_{-\infty}^{\infty}\left(\frac{E-E^{\prime}}{\tilde{\gamma}}\right)^{k} \frac{\exp \left[-\left(E-E^{\prime}\right)^{2} / \tilde{\gamma}^{2}\right]}{\left(\Gamma_{m} / 2\right)^{2}+\left(E_{m}-E^{\prime}\right)^{2}} \mathrm{~d} E^{\prime}=\frac{2 \pi(-1)^{k}}{\Gamma_{m}} \operatorname{Re}\left\{\frac{d^{k / 2}}{d \lambda^{k / 2}} w\left[\frac{\sqrt{\lambda}}{\tilde{\gamma}}\left(E_{m}+i \frac{\Gamma_{m}}{2}-E\right)\right]\right\}_{\lambda=1}
$$

with $k=0,2, \ldots, 2 s$. The final expressions for $\widetilde{g}(E)$ obtained in this way for $s \leq 5$ are given in Table I.

\begin{tabular}{|l|l|}
\hline \hline$s$ & $\tilde{\gamma} \sqrt{\pi} \widetilde{g}(E)$ \\
\hline \hline 0 & $\sum_{m} \Re e w\left(z_{m}\right)$ \\
\hline 1 & $\sum_{m} \Re e\left(L_{1}^{\frac{1}{2}}\left(z_{m}^{2}\right) w\left(z_{m}\right)+i z_{m} / \sqrt{\pi}\right)$ \\
\hline 2 & $\sum_{m} \Re e\left(L_{2}^{\frac{1}{2}}\left(z_{m}^{2}\right) w\left(z_{m}\right)-i z_{m}\left(2 z_{m}^{2}-9\right) / 4 \sqrt{\pi}\right)$ \\
\hline 3 & $\sum_{m} \Re e\left(L_{3}^{\frac{1}{2}}\left(z_{m}^{2}\right) w\left(z_{m}\right)+i z_{m}\left(4 z_{m}^{4}-40 z_{m}^{2}+87\right) / 24 \sqrt{\pi}\right)$ \\
\hline 4 & $\sum_{m} \Re e\left(L_{4}^{\frac{1}{2}}\left(z_{m}^{2}\right) w\left(z_{m}\right)-i z_{m}\left(8 z_{m}^{6}-140 z_{m}^{4}+690 z_{m}^{2}-975\right) / 192 \sqrt{\pi}\right)$ \\
\hline 5 & $\sum_{m} \Re e\left(L_{5}^{\frac{1}{2}}\left(z_{m}^{2}\right) w\left(z_{m}\right)+i z_{m}\left(16 z_{m}^{8}-432 z_{m}^{6}+3752 z_{m}^{4}-12180 z_{m}^{2}+12645\right) / 1920 \sqrt{\pi}\right)$ \\
\hline \hline
\end{tabular}

TABLE I. Strutinsky-averaged density of states (21) for orders $0 \leq s \leq 5$ of the correction polynomial. The definitions of $z_{m}$ and $w(z)$ are given in Eq. (8) with $\gamma$ replaced by $\tilde{\gamma}$. 


\section{REFERENCES}

[1] E. Beth and G. E. Uhlenbeck, Physica 4, 915 (1937).

[2] R. Feynman: The Feynman Lectures on Physics, Vol. I (Addison-Wesley, 1964), chapters 23 and 24.

[3] A. Bäcker, A. Manze, B. Huckestein and R. Ketzmerick, Phys. Rev. E 66, 016211 (2002).

[4] M. C. Gutzwiller, J. Math. Phys. 12, 343 (1971), and earlier references quoted therein.

[5] M. L. Du and J. B. Delos, Phys. Rev. A 38, 1896 (1988); ibid., 1913 (1988).

[6] M. C. Gutzwiller: Chaos in Classical and Quantum Mechanics (Springer, New York, 1990).

[7] H.-J. Stöckmann: Quantum Chaos: An Introduction (Cambridge Univ. Press, 1999).

[8] F. Haake: Quantum signatures of chaos (Springer, Berlin, 2001).

[9] P. Gaspard and S. A. Rice, J. Chem. Phys. 90, 2242 (1989).

[10] A. Wirzba, Phys. Rep. 309, 1 (1999).

[11] G. Tanner, K. T. Hansen, and J. Main, Nonlinearity 9, 1641 (1996).

[12] V. Kondratovich and J. B. Delos, Phys. Rev. A 57, 4654 (1998).

[13] T. Bartsch, Ph.D. thesis (University of Stuttgart, 2002).

[14] A. M. Ozorio de Almeida and J. H. Hannay, J. Phys. A 20, 5873 (1987).

[15] M. Sieber, J. Phys. A 29, 4715 (1996);

H. Schomerus and M. Sieber, J. Phys. A 30, 4537 (1997);

M. Sieber and H. Schomerus, J. Phys. A 31, 165 (1998).

[16] H. Schomerus, Europhys. Lett. 38, 423 (1997);

H. Schomerus and F. Haake, Phys. Rev. Lett. 79, 1022 (1997).

[17] H. Schomerus, J. Phys. A 31, 4167 (1998).

[18] J. Kaidel and M. Brack, Phys. Rev. E 70, 016206 (2004).

[19] M. Hénon and C. Heiles, Astr. J. 69, 73 (1964).

[20] G. H. Walker and J. Ford, Phys. Rev. 188, 416 (1969).

[21] A. J. Lichtenberg and M. A. Liebermann, Regular and Stochastic Motion (Springer, New York, 1981).

[22] M. Brack, J. Blaschke, S. C. Creagh, A. G. Magner, P. Meier, and S. M. Reimann, Z. Phys. D 40, 276 (1997); L. Christensson, H. Linke, P. Omling, P. E. Lindelof, I. V. Zozoulenko and K.-F. Berggren, Phys. Rev. B 57, 19 (1998).

[23] N. Pomphrey, J. Phys. B 7, 1909 (1974).

[24] K. Efstathiou and D. A. Sadovski, Nonlinearity 17, 415 (2004).

[25] V. M. Strutinsky and A. G. Magner, Sov. J. Part. Nucl. 7, 138 (1976) [Elem. Part. \& Nucl. (Atomizdat, Moscow) 7, 356 (1976)].

[26] V. M. Strutinsky, Nucl. Phys. A 95, 420 (1967); ibid. A 122, 1 (1968).

[27] M. Brack and R. K. Bhaduri, Semiclassical Physics (Westview Press, Boulder, 2003).

[28] M. Brack, P. Meier, and K. Tanaka, J. Phys. A 32, 331 (1999); see also earlier references quoted therein.

[29] M. Abramowitz and I. A. Stegun: Handbook of Mathematical Functions (Dover, New York, 1965), equation 7.4.13.

[30] E. Balslev and J. C. Combes, Commun. Math. Phys. 22, 280 (1971).

[31] W. P. Reinhardt, Ann. Rev. Phys. Chem. 33, 223 (1982); see also B. Simon, Ann. Math. 97, 247 (1973).

[32] R. Yaris and P. Winkler, J. Phys. B 11, 1475 (1978); P. Winkler and R. Yaris, J. Phys. B 11, 1481 (1978); ibid. B 11, 4257 (1978).

[33] N. Moiseyev, Phys. Rep. 302, 211 (1998).

[34] R. Yaris, J. Bendler, R. A. Lovett, C. M. Bender, and P. A. Fedders, Phys. Rev. A 18 (1978) 5; L. S. Cederbaum and P. Winkler, Theor. Chim. Acta 88, 257 (1994).

[35] B. A. Waite and W. H. Miller, J. Chem. Phys. 74, 7 (1981).

[36] M. Brack, Ph.D. thesis (University of Basel, 1972): see M. Brack and H.-C. Pauli, Nucl. Phys. A 207, 401 (1973).

[37] C. K. Ross and R. K. Bhaduri, Nucl. Phys. A 188, 566 (1972).

[38] A. Sobiczewski, A. Gyurkovich, and M. Brack, Nucl. Phys. A 289, 346 (1977).

[39] J. Kaidel, Ph.D. thesis (University of Regensburg, 2003): 〈http://www.opus-bayern.de/uni-regensburg/volltexte/ $2004 / 349\rangle$.

[40] R. C. Churchill, G. Pecelli, and D. L. Rod, in: Stochastic Behaviour in Classical and Quantum Hamiltonian Systems, ed. by G. Casati and J. Ford (Springer, New York, 1979) p. 76.

[41] K. T. R. Davies, T. E. Huston, and M. Baranger, Chaos 2 (1992) 215.

[42] W. M. Vieira and A. M. Ozorio de Almeida, Physica D 90, 9 (1996).

[43] M. Brack, Foundations of Physics 31, 209 (2001).

[44] M. Brack, M. Mehta, and K. Tanaka, J. Phys. A 34, 8199 (2001).

[45] S. C. Creagh, J. M. Robbins, and R. G. Littlejohn, Phys. Rev. A 42, 1907 (1990). 